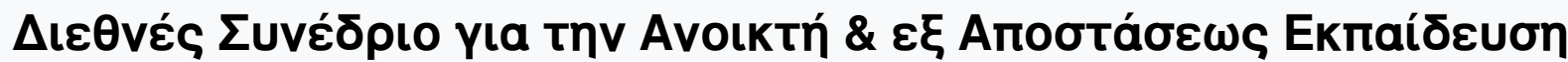

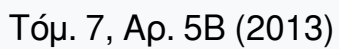

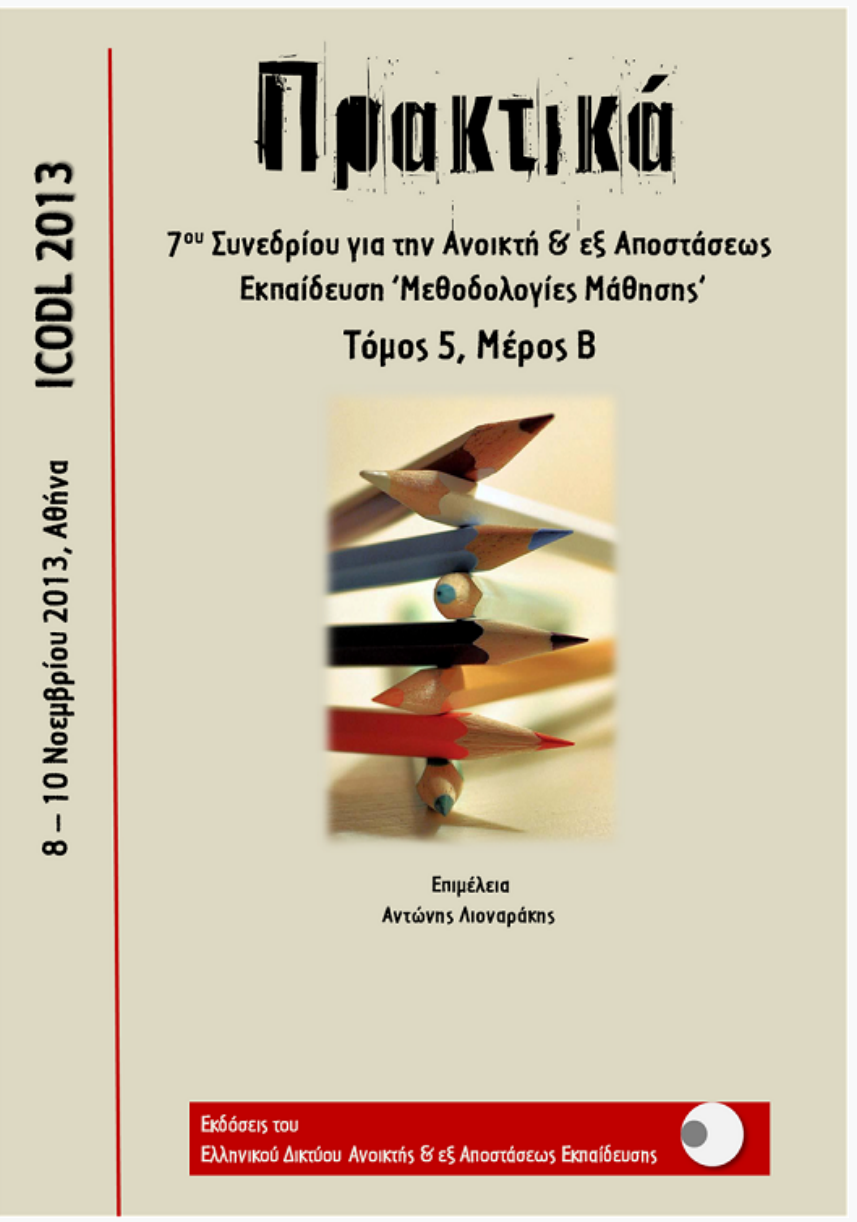

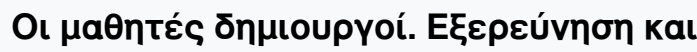

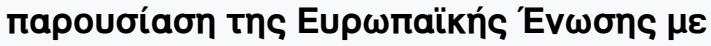

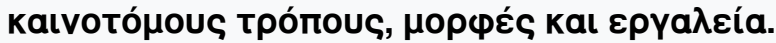

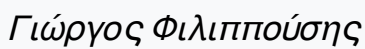

doi: $10.12681 /$ icodl. 558 


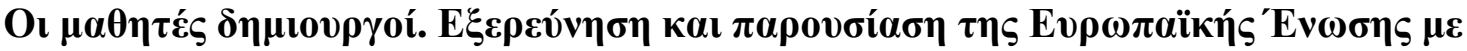

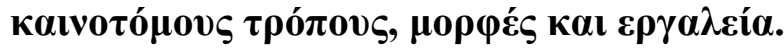

\section{The Students creators. Exploration and presentation of the European Union in innovative ways, styles and tools}

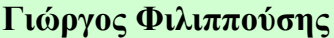

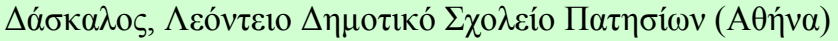

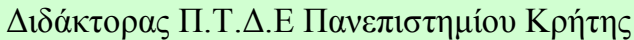 \\ filvisg@outlook.com
}

\begin{abstract}
This innovative educational activity takes place in the framework of the program «Teachers 4 Europe». The program «Teachers 4 Europe» has seen designed to inform learners about European matters and support their innovative teaching methods, which could also benefit from technology and communications (www.teachers4europe.gr).

The purpose of this study is to present and inform about the role of the European Union in ways, forms and tools that pupils of E' Primary School choose. In the main feature is the integration and utilization of new technologies in educational and social conditions that may encourage the cultivation of critical thinking and contribute to the creation of

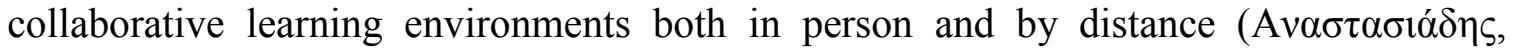
2006).

The aims of the program are multidimensional and begin to help teachers and students familiarize with ICT and with the use of those tools in pedagogical conditions for the benefit of the learning process. This would help building the knowledge, not only face to face, but also from distance through collaborative environments, and teach the growing interest of students for the European Union affairs, culture, history, language and respect for diversity, cooperation and solidarity.

It is important to underline that that teachers and students are the creators (co-creators) of educational materials in many differentiated and innovative ways.

Note: All the action and activities can be found at

http://www.citizens-europe.leonteios.edu.gr/.

\section{Пєрí $\lambda \psi \eta$}

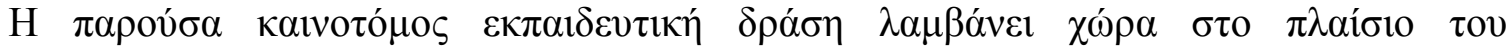

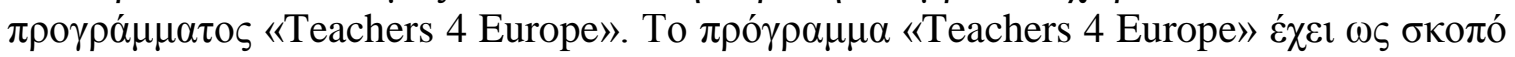

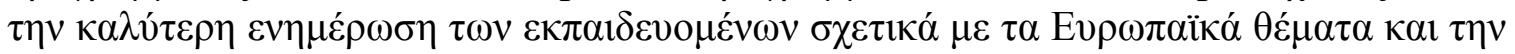

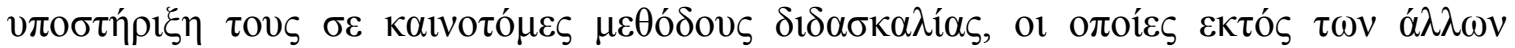

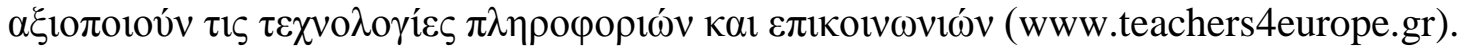

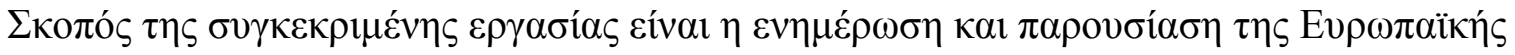

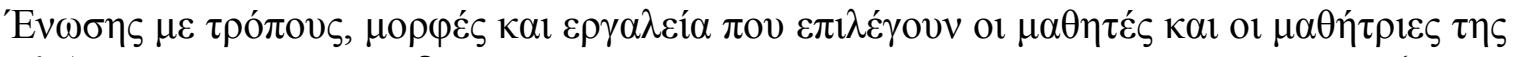

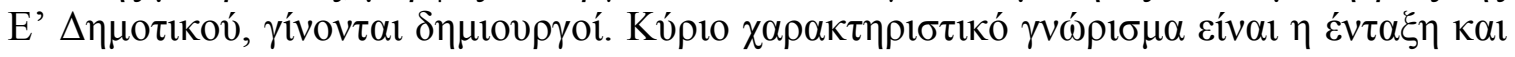

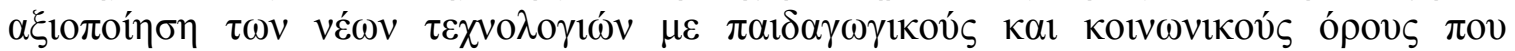

SECTION B: applications, experiences, good practices, descriptions and outlines, educational activities, issues for dialog and discussion 


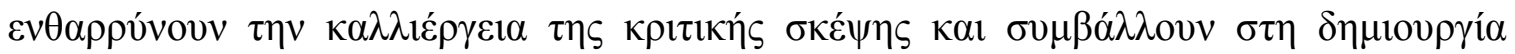

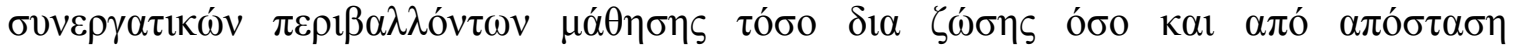

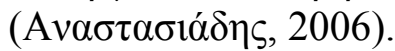

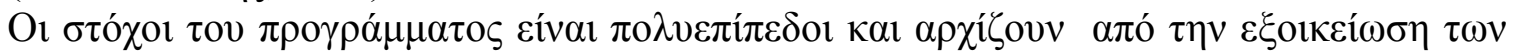

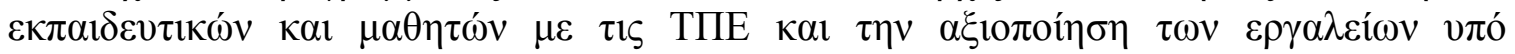

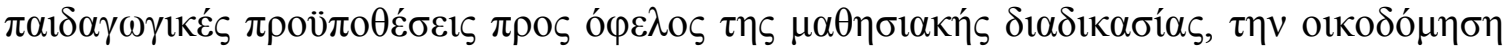

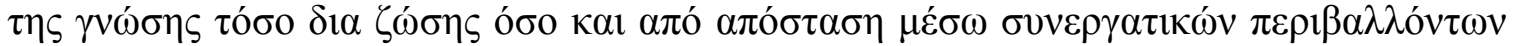

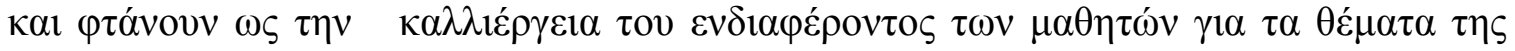

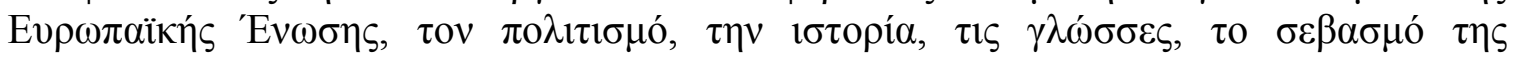

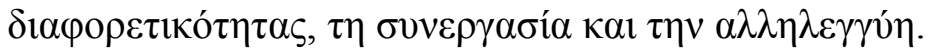

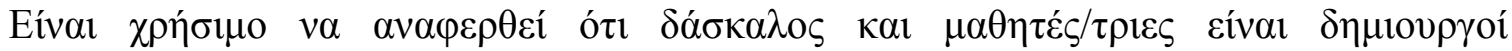

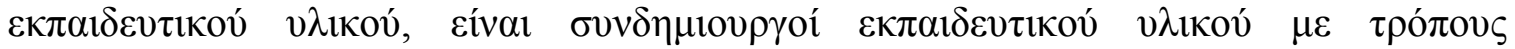

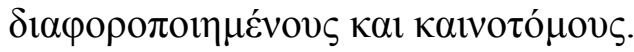

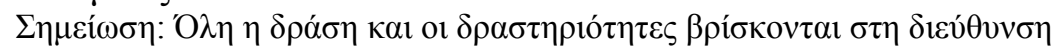
http://www.citizens-europe.leonteios.edu.gr/.

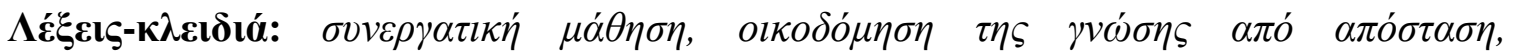

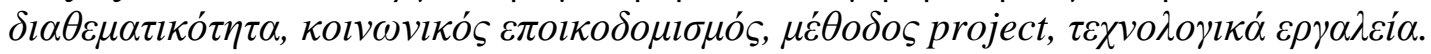

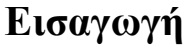

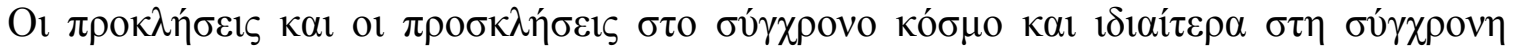

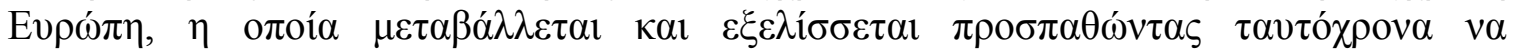

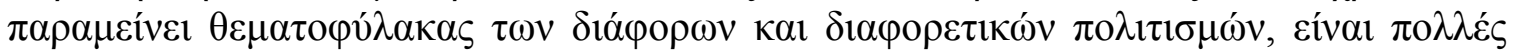

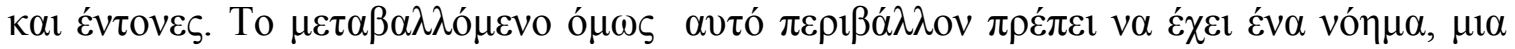

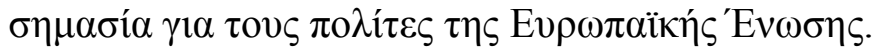

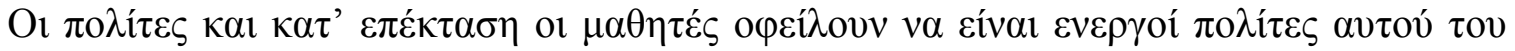

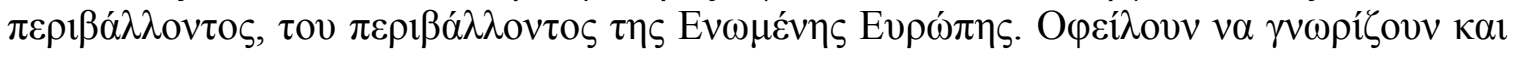

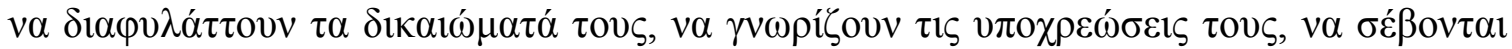

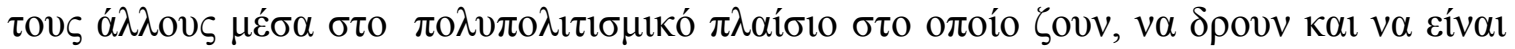

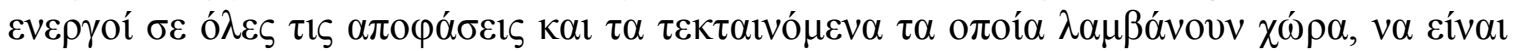

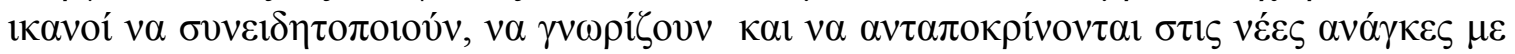

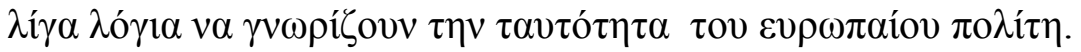

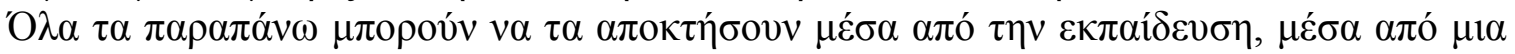

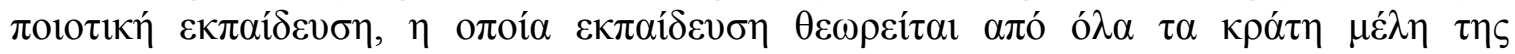

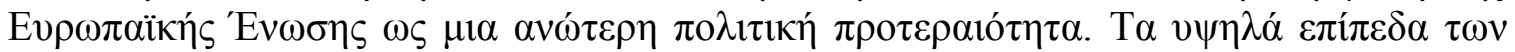

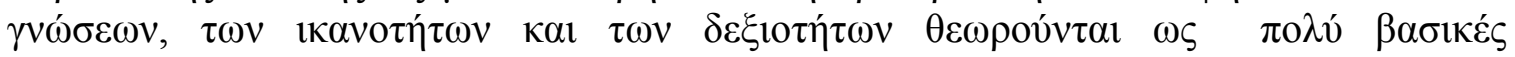

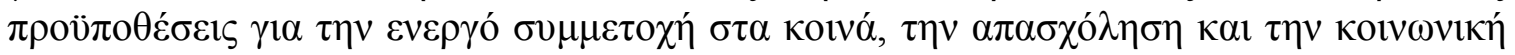

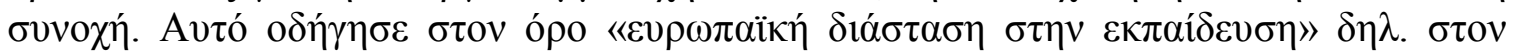

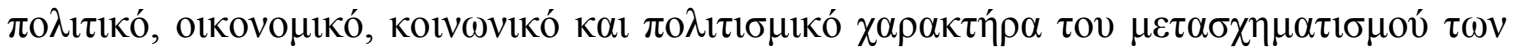

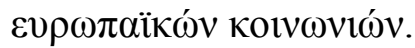

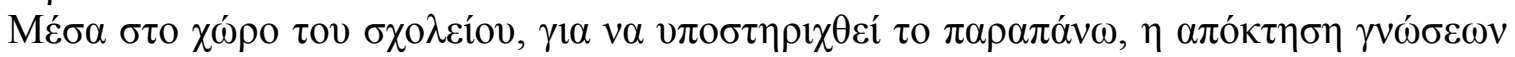

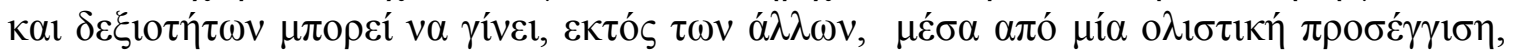

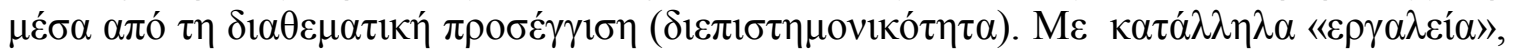

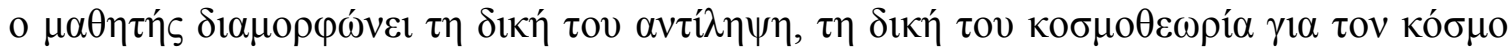

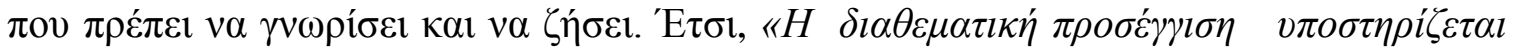

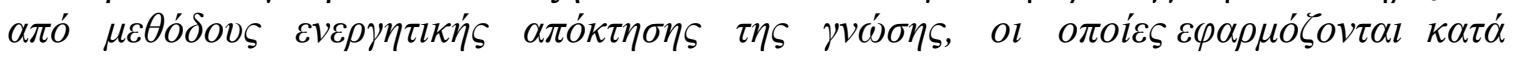

SECTION B: applications, experiences, good practices, descriptions and outlines, educational activities, issues for dialog and discussion 


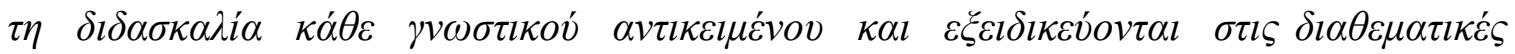

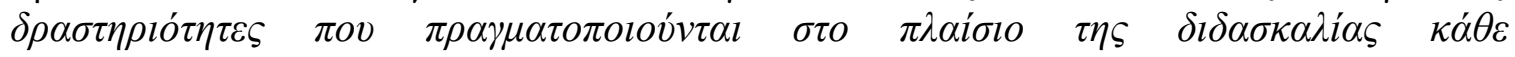

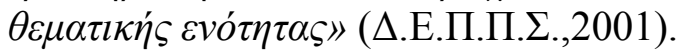

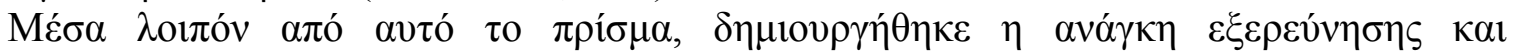

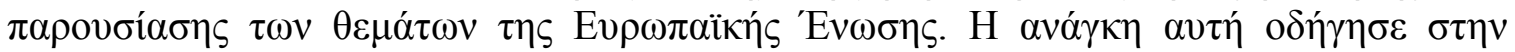

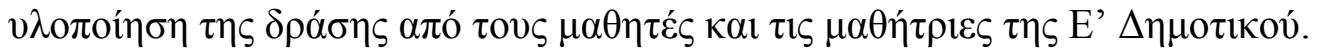

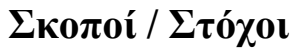

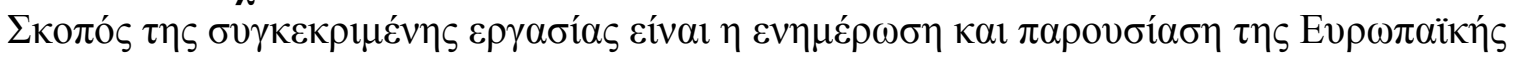

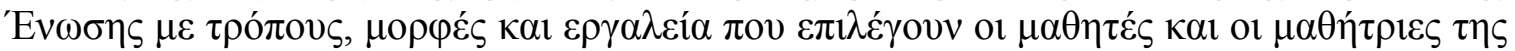

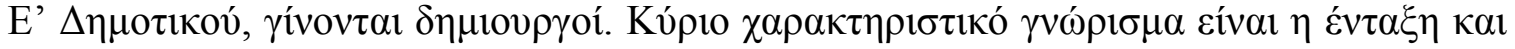

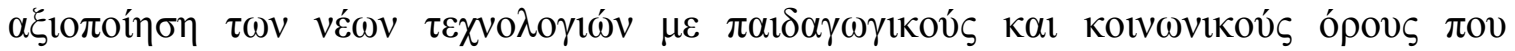

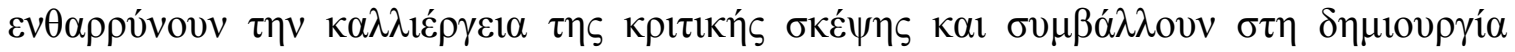

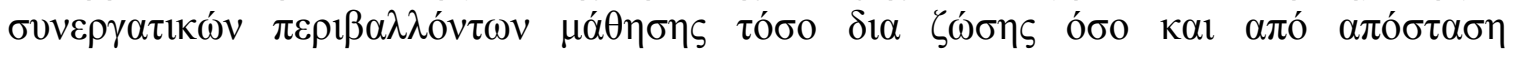

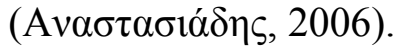

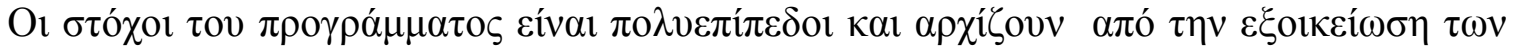

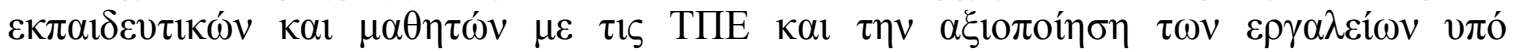

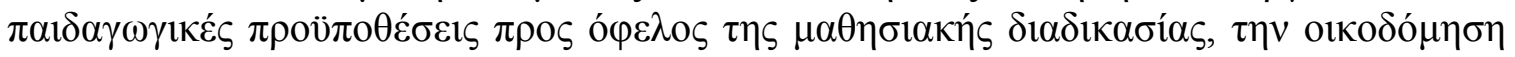

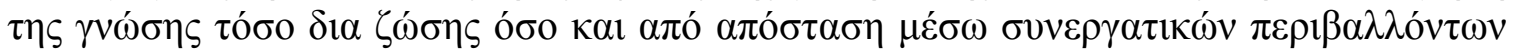

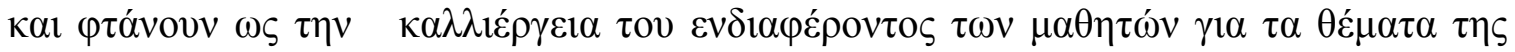

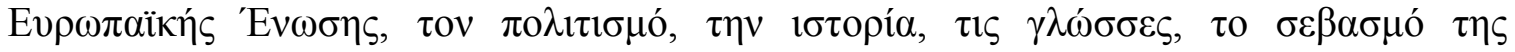

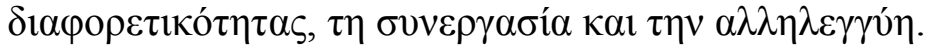

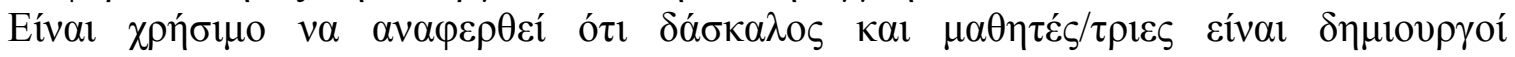

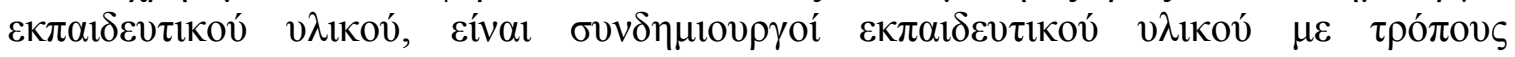

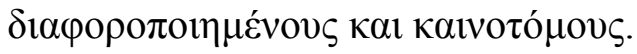

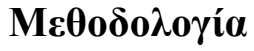

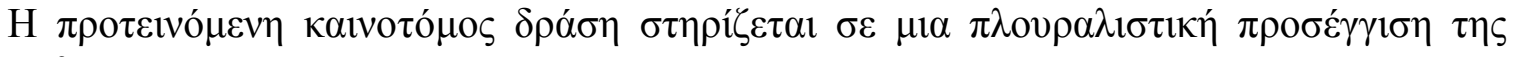
$\mu \alpha ́ \theta \eta \sigma \eta \varsigma$.

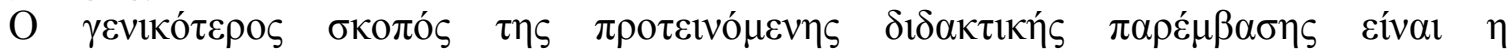

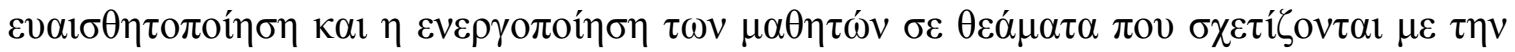

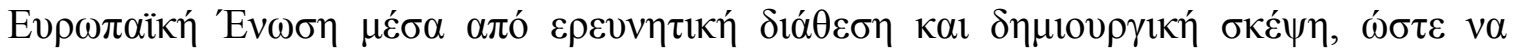

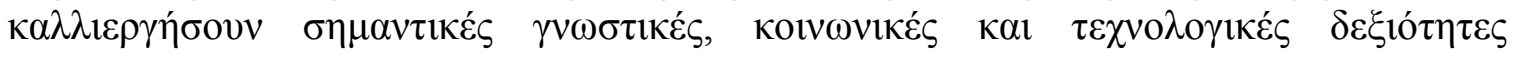
(Anastasiades,2009).

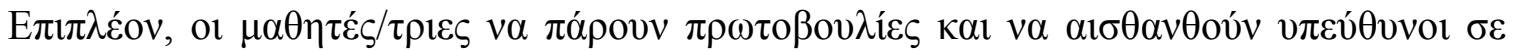

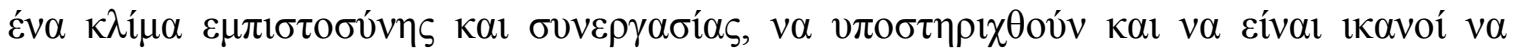

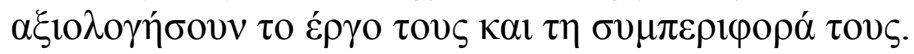

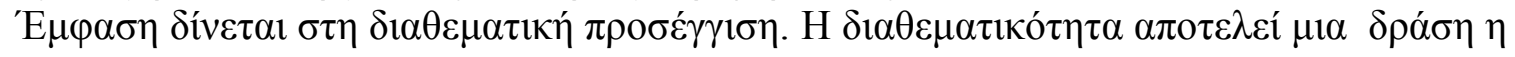

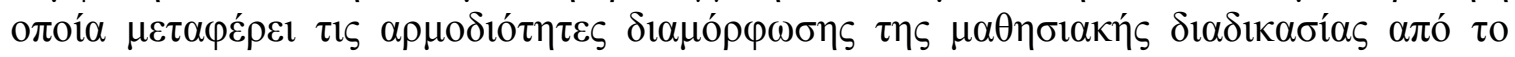

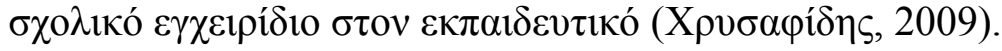

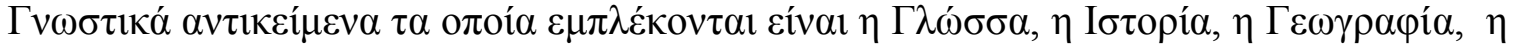

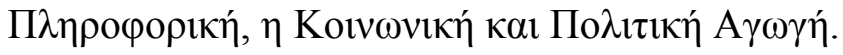

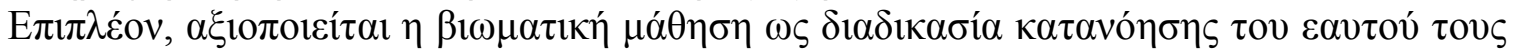

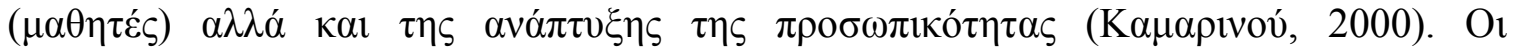

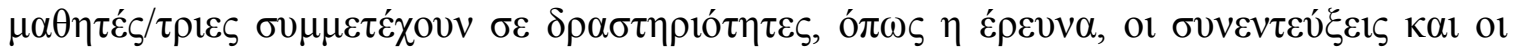

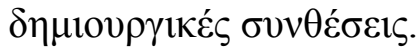




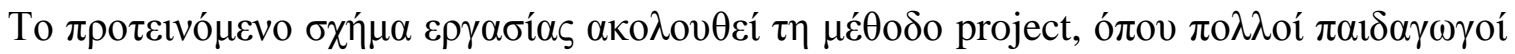

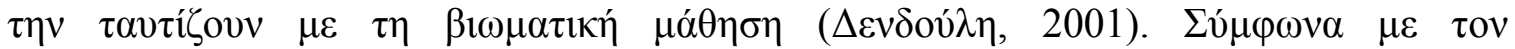

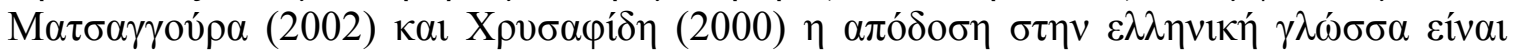

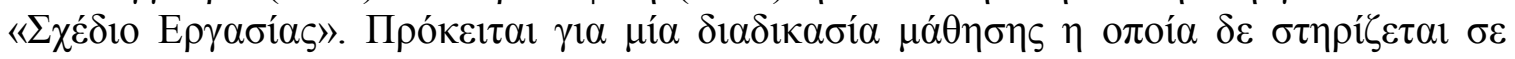

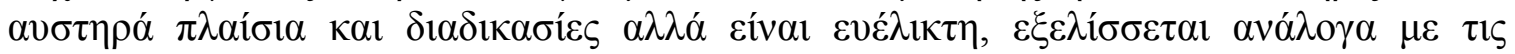

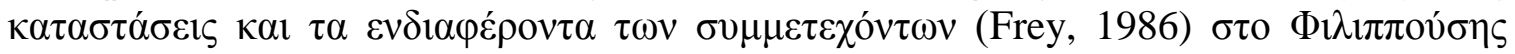
(2012) .

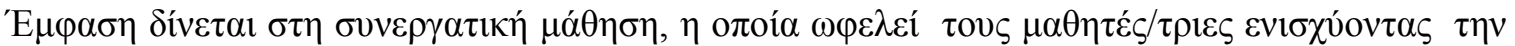

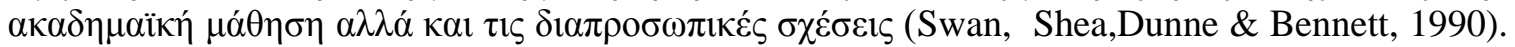

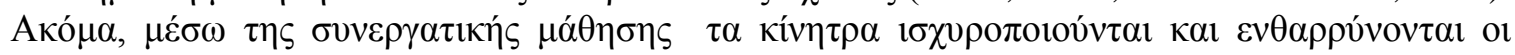

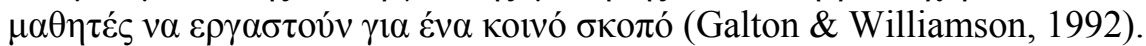

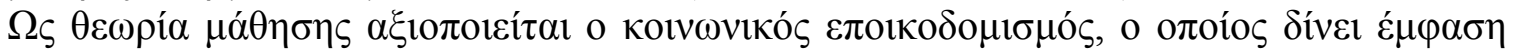

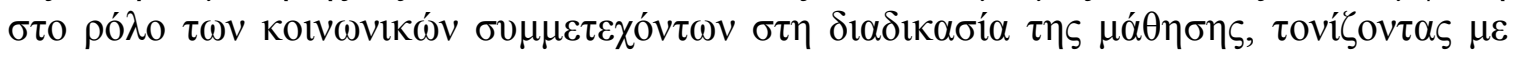

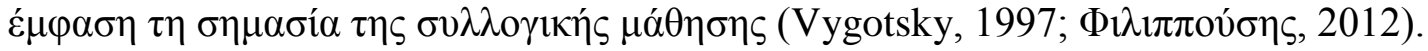

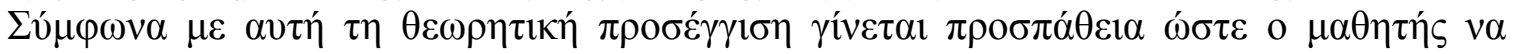

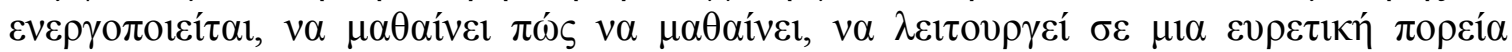

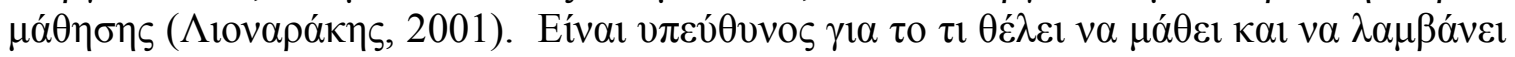

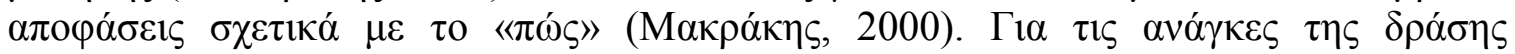

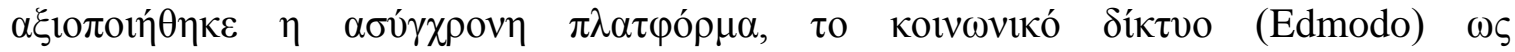

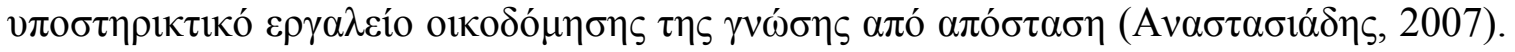

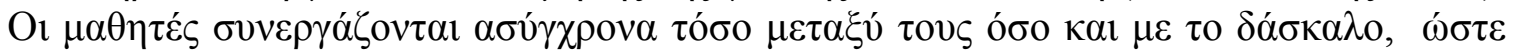

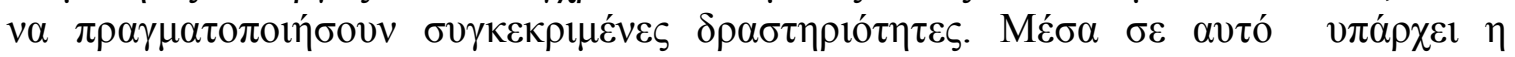

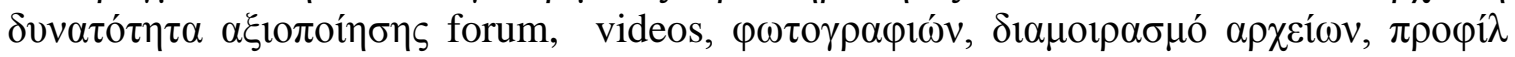

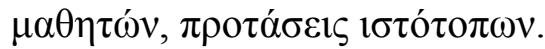

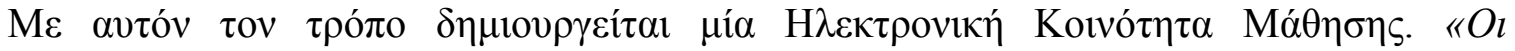

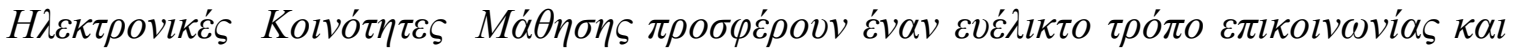

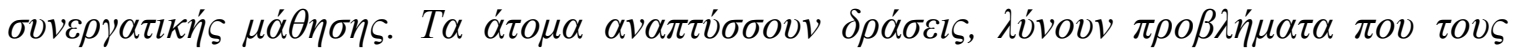

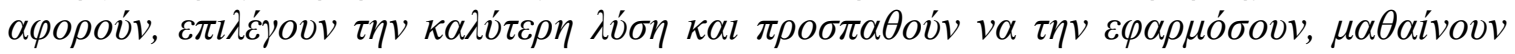

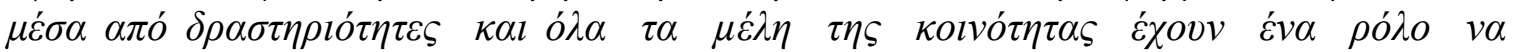

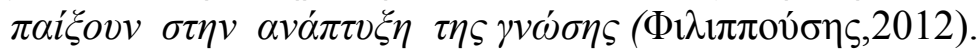

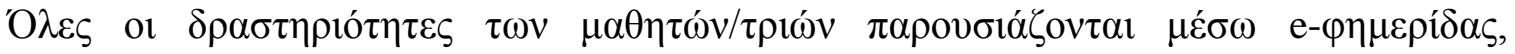

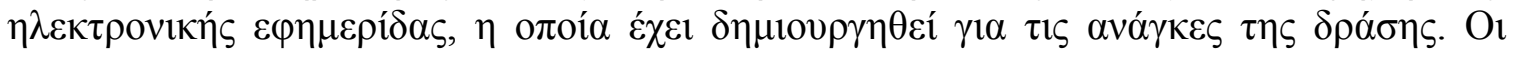

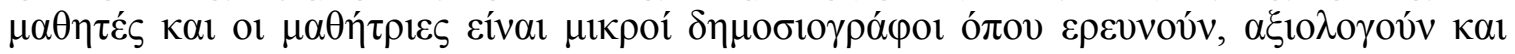
$\pi \alpha \rho o v \sigma i a ́ \zeta o v v$.

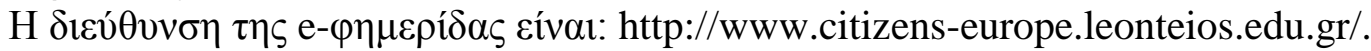

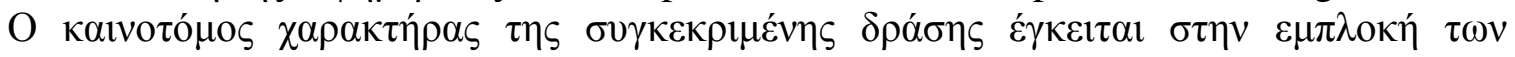

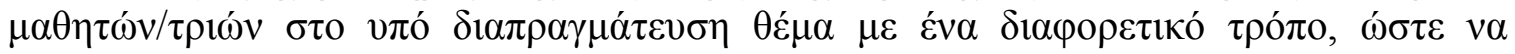

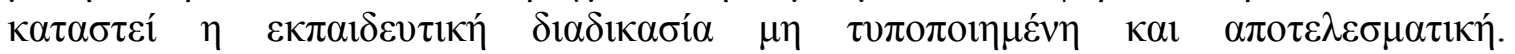

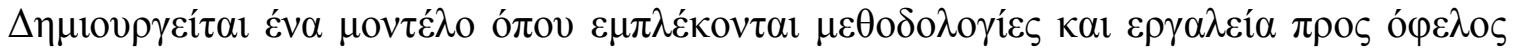

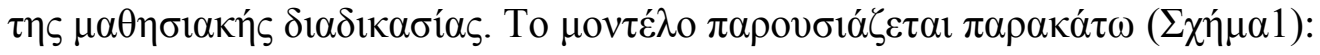




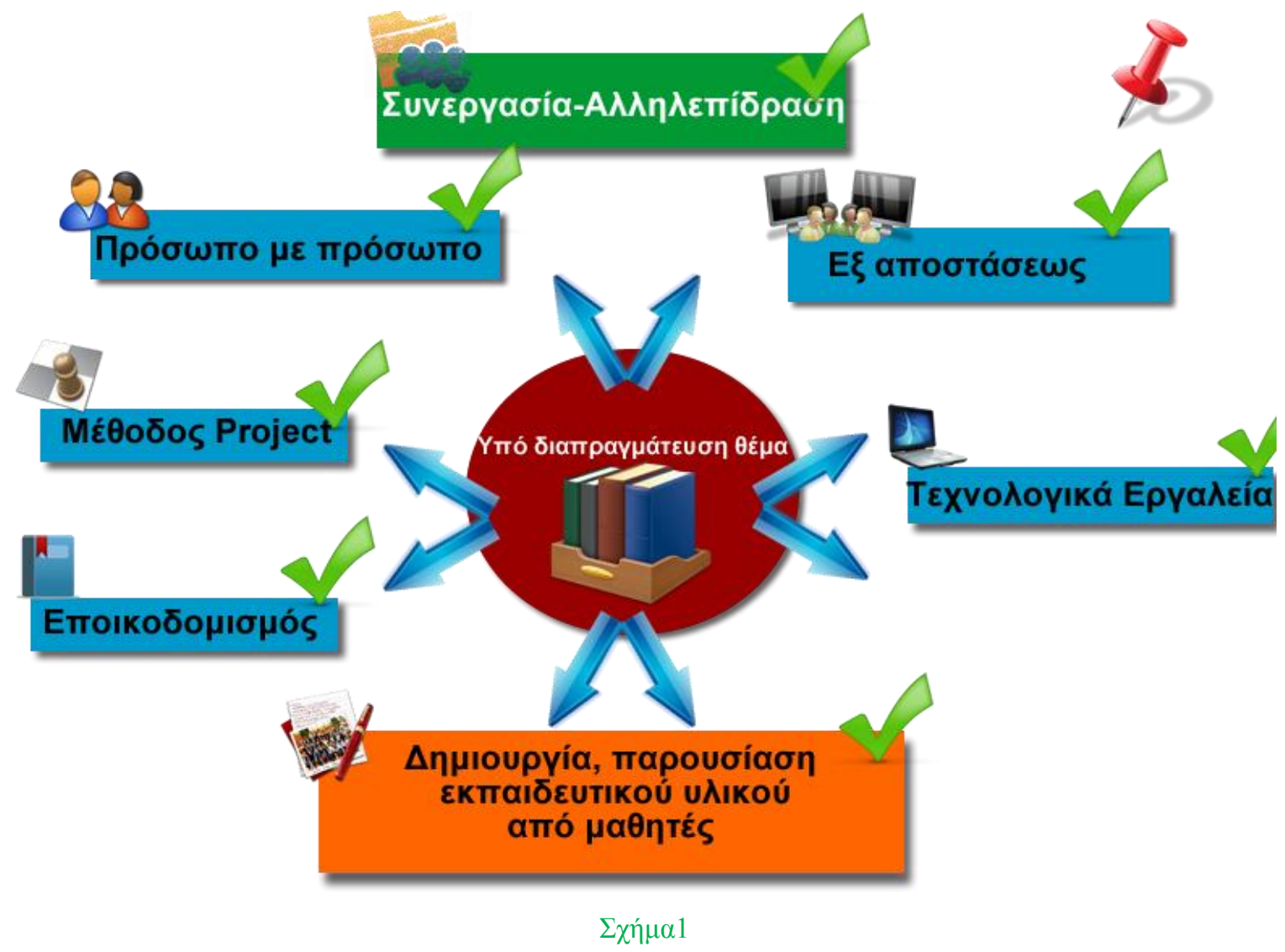

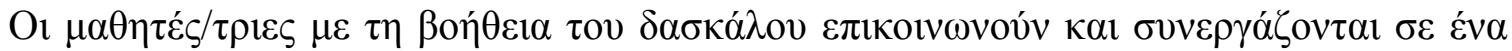

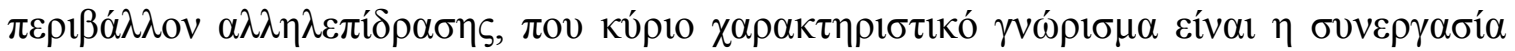

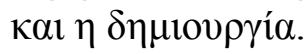

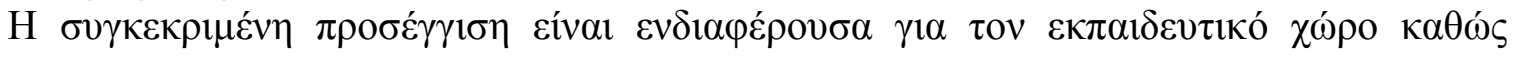

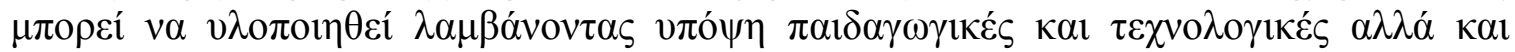

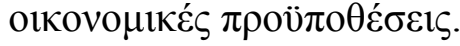

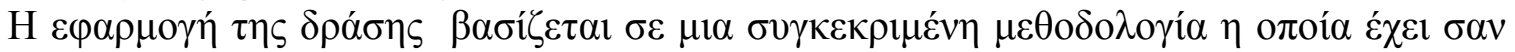

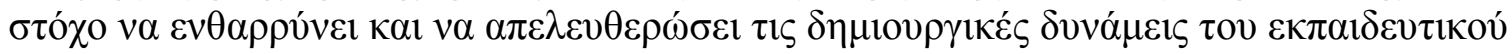

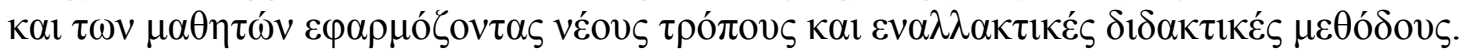

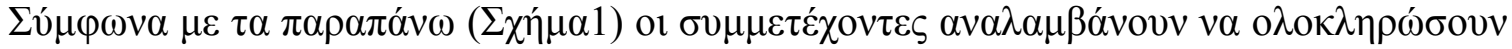

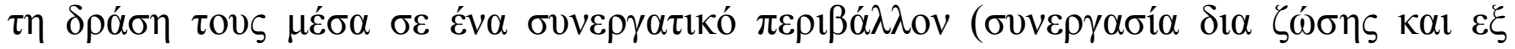

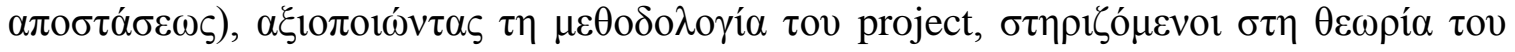

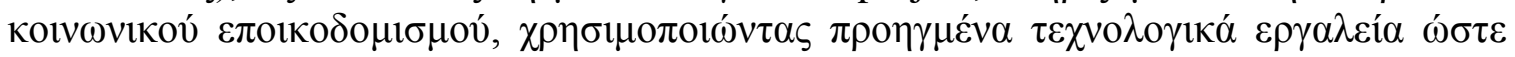

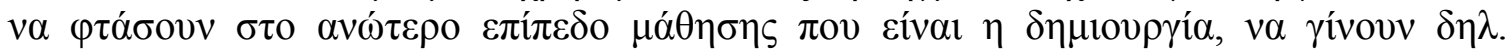

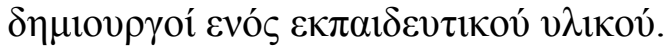

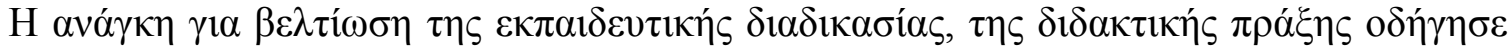

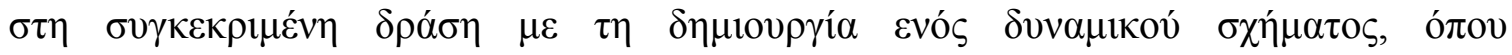

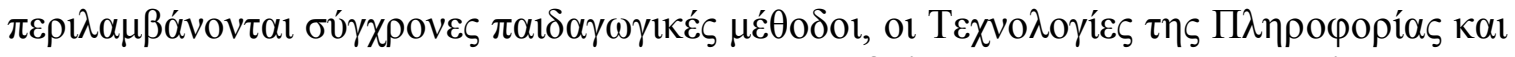

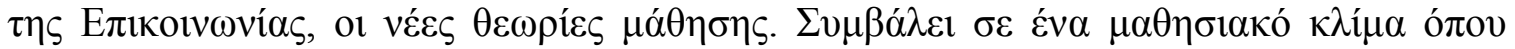

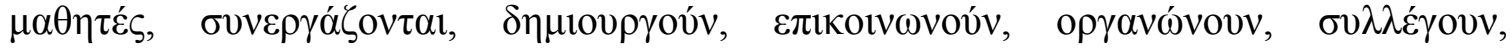

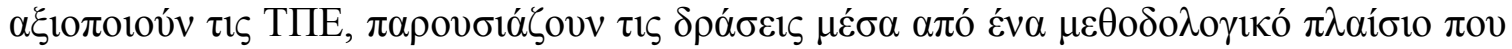

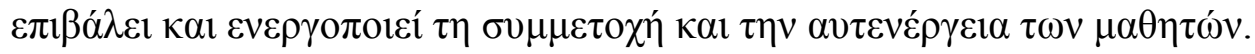




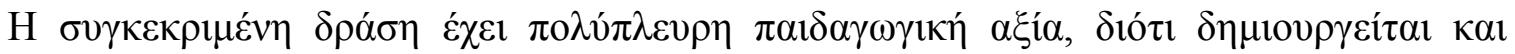

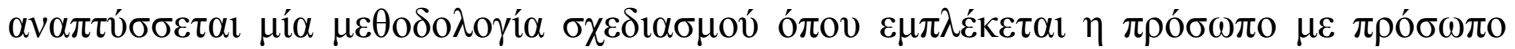

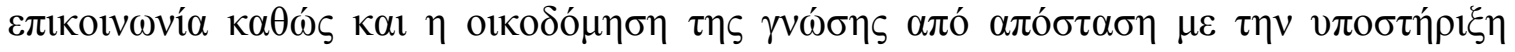

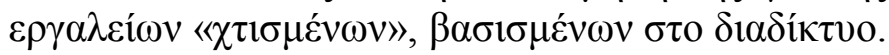

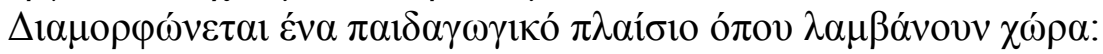

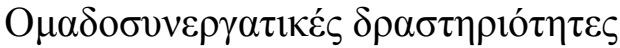

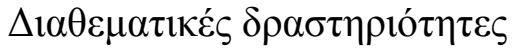

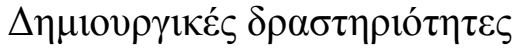

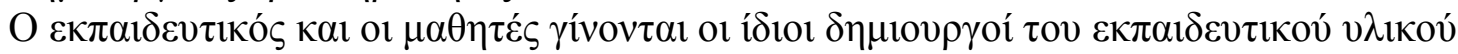

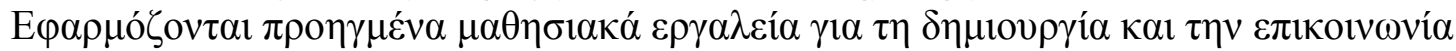

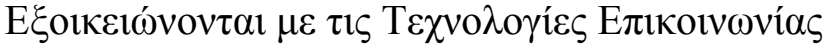

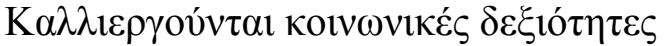

$\Omega \varphi \varepsilon \lambda \circ v^{\prime} \tau \alpha \imath \mu \alpha \theta \eta \sigma \imath \alpha \kappa \alpha ́$

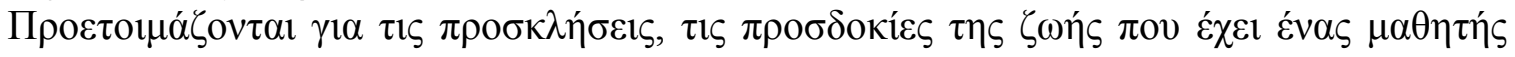

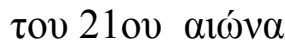

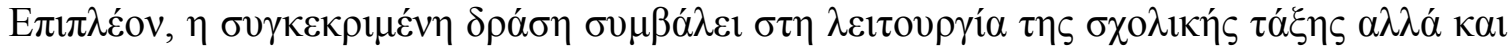

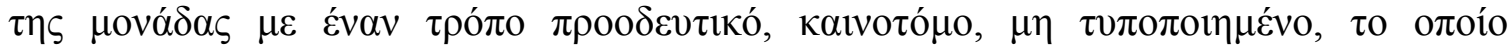

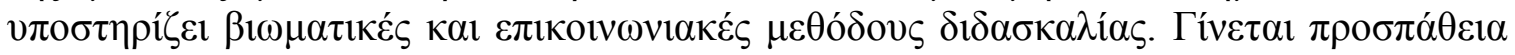

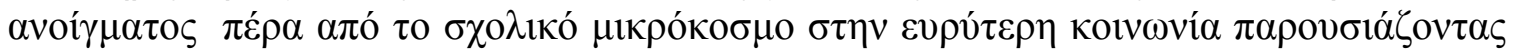

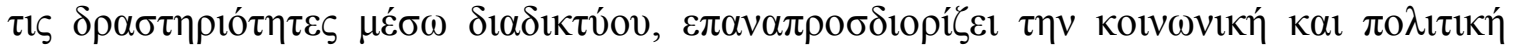

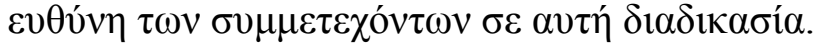

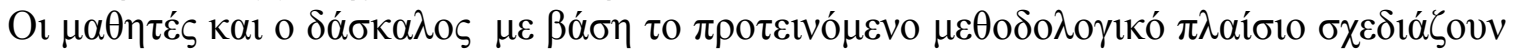

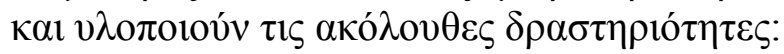

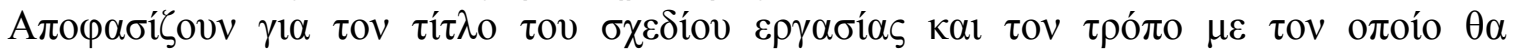

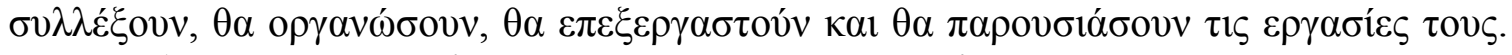

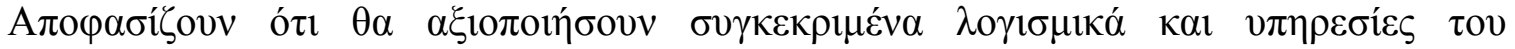
$\delta 1 \alpha \delta 1 \kappa \tau v ́ o v$.

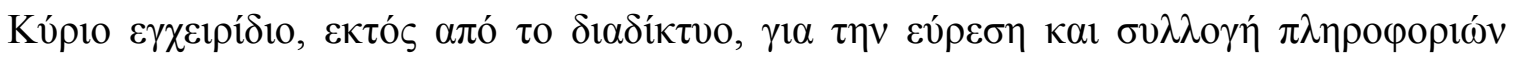

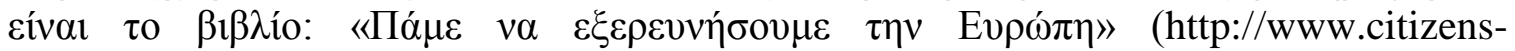
europe.leonteios.edu.gr//files/aformisi/ee_book/index.html).

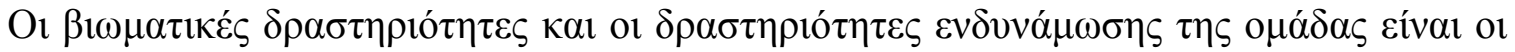

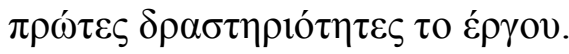

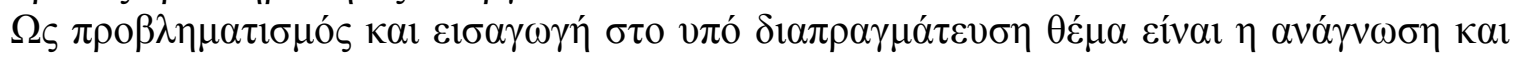

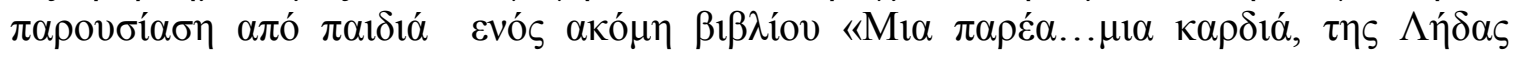

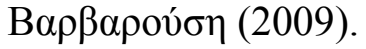

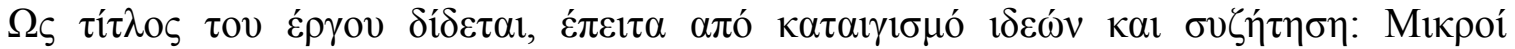

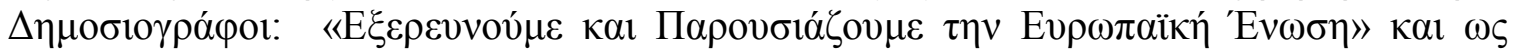

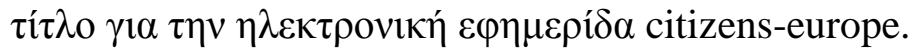

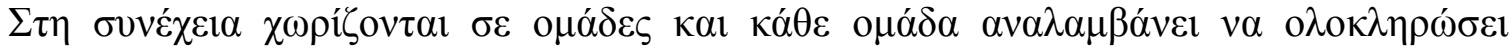

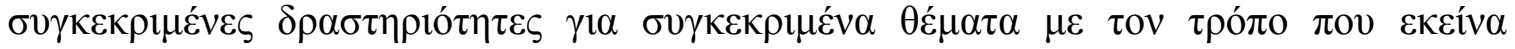
$\varepsilon \pi \imath \theta u \mu o v ́ v$.

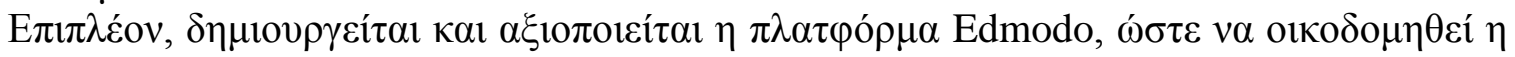

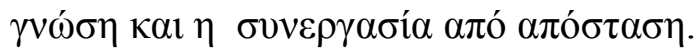

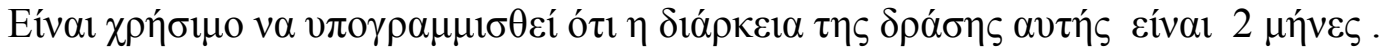

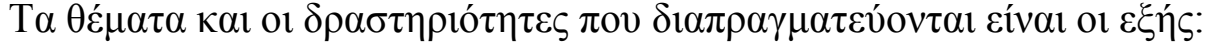

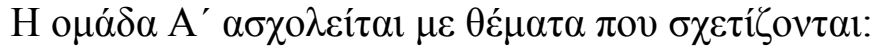




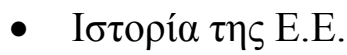

- $\Sigma \eta \mu \alpha \nu \tau \iota \kappa \varepsilon ́ \varsigma \pi \rho о \sigma \omega \pi \imath \kappa o ́ \tau \eta \tau \varepsilon \varsigma \tau \eta \varsigma$ E.E.

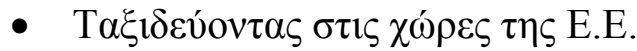

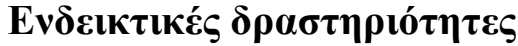

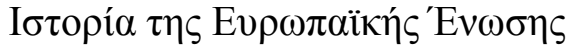

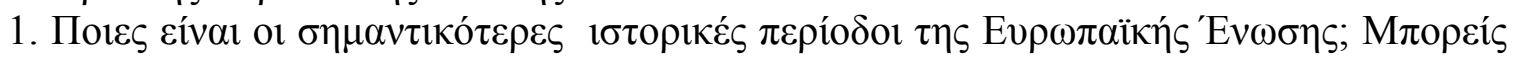

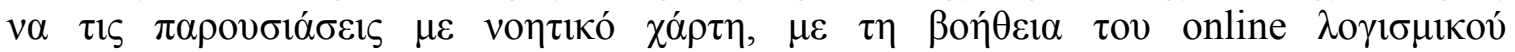

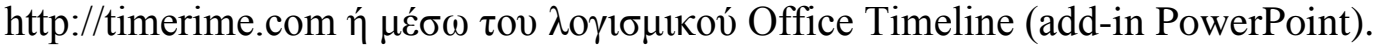

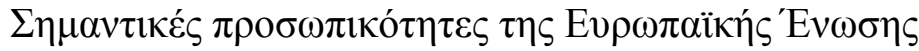

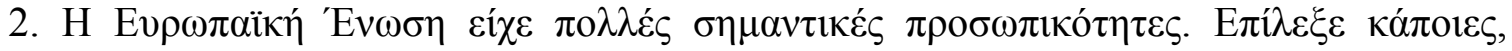

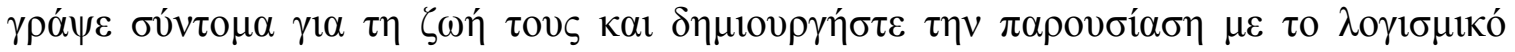
Deepzoom $\tau \eta \varsigma$ Microsoft.

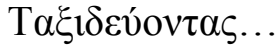

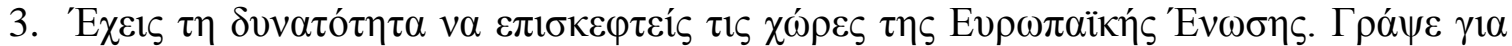

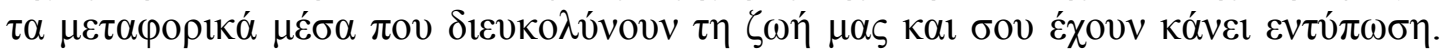

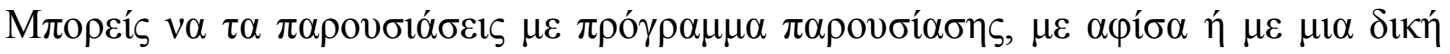

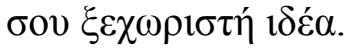

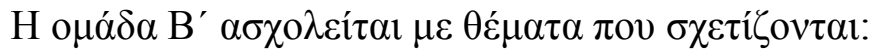

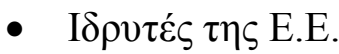

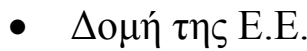

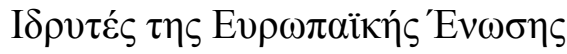

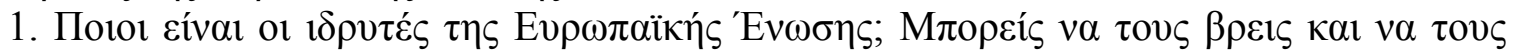

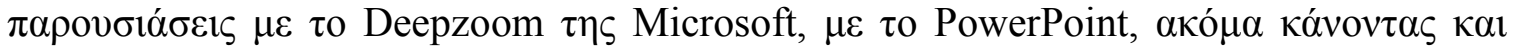

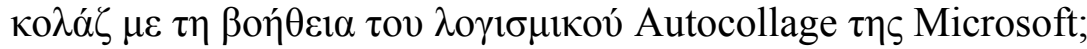

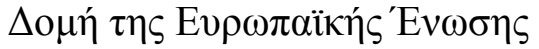

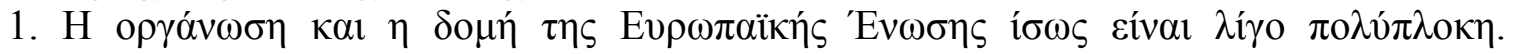

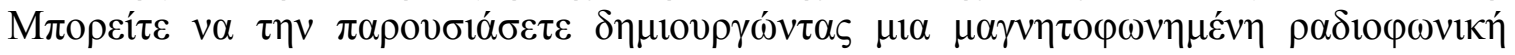

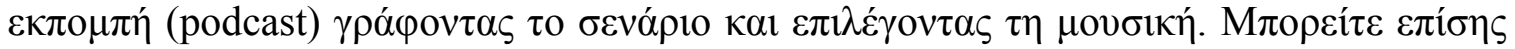

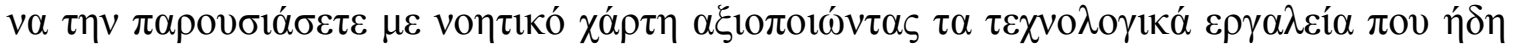
$\gamma \vee \omega \rho i ́ \zeta \varepsilon \tau \varepsilon$.

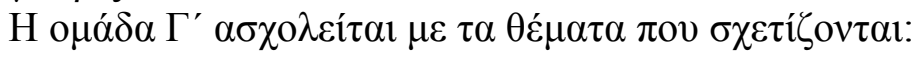

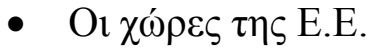

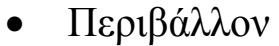

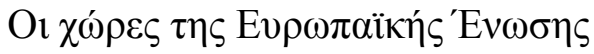

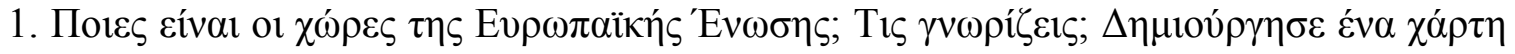

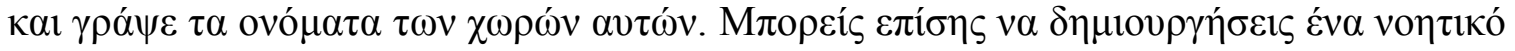

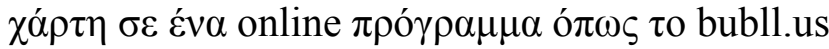

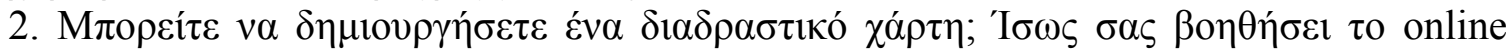

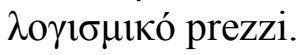

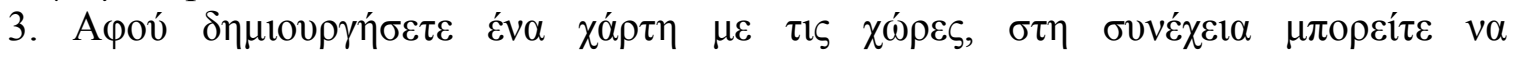
$\delta \eta \mu \iota v \rho \gamma \eta ́ \sigma \varepsilon \tau \varepsilon$ puzzles.

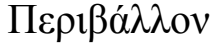




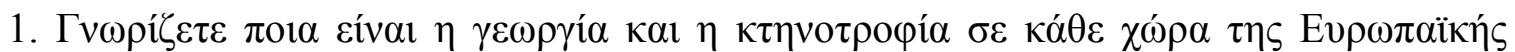

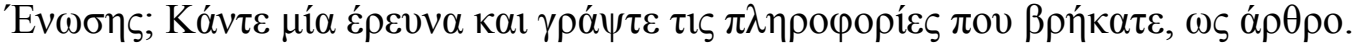

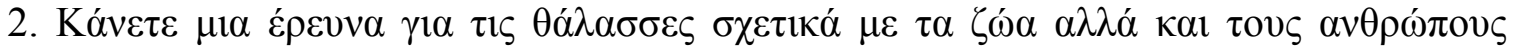

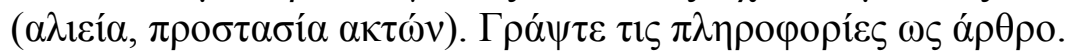

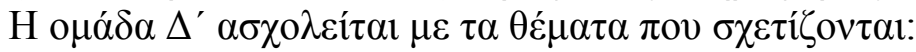

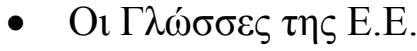

- $\quad \Sigma v ́ \mu \beta o \lambda \alpha \tau \eta \varsigma$ E.E

- $\mathrm{K} \lambda i ́ \mu \alpha$

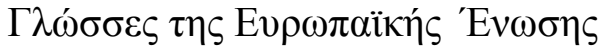

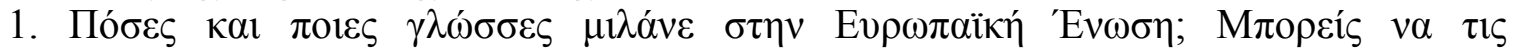

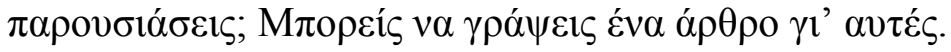

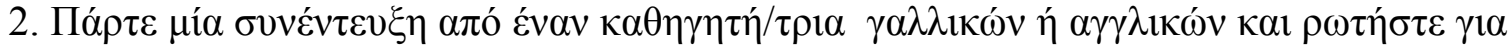

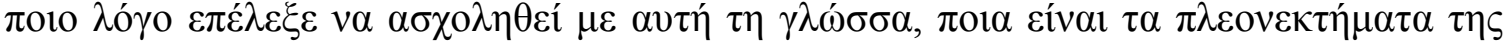

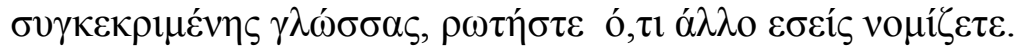

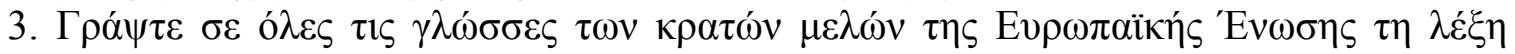

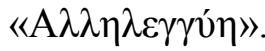

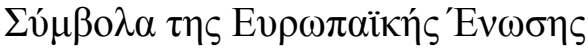

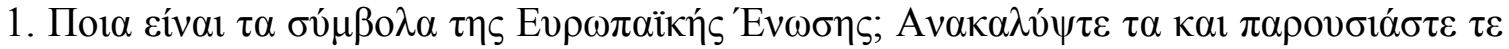

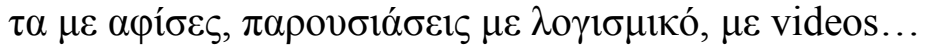

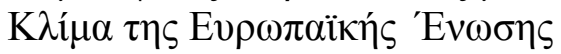

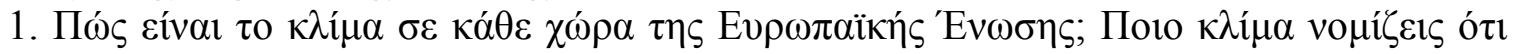

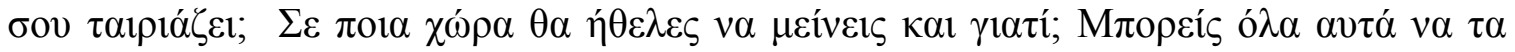

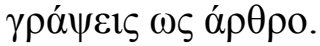

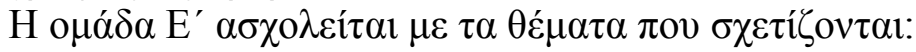

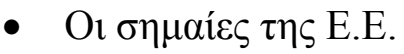

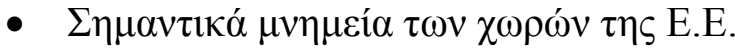

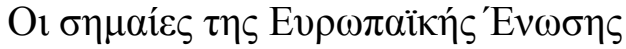

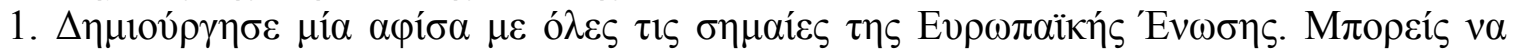

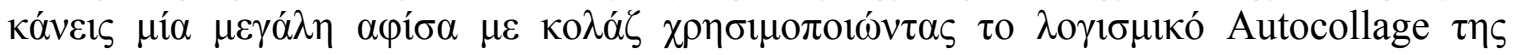
Microsoft

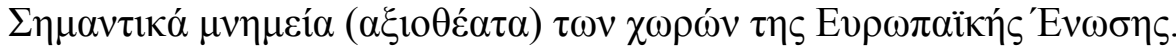

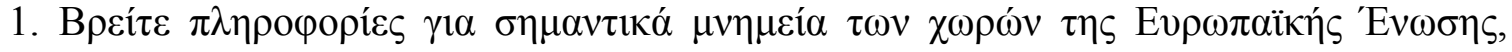

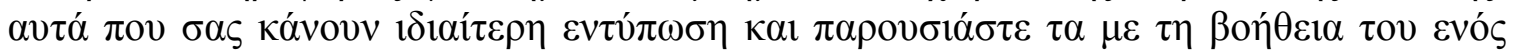

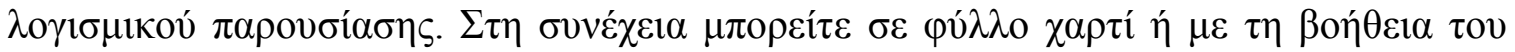

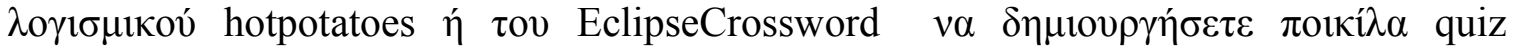
( $\sigma \tau \alpha \cup \rho o ́ \lambda \varepsilon \xi \alpha, \sigma v \mu \pi \lambda \eta ́ \rho \omega \sigma \eta \varsigma \kappa \varepsilon v o v ́, \varepsilon \rho \omega \tau \eta ́ \sigma \varepsilon 1 \varsigma \kappa . \alpha$.

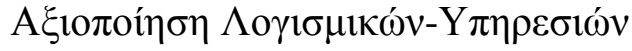

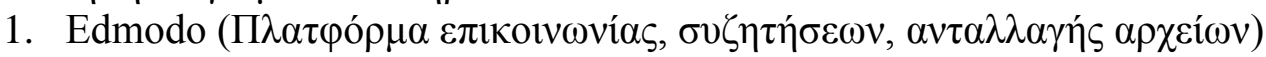

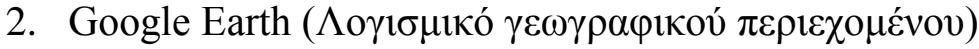

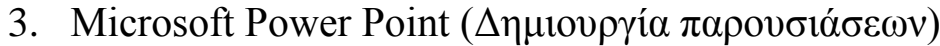

4. Prezzi - http://prezi.com $(\Delta \eta \mu \iota 0 v \rho \gamma i ́ \alpha \pi \alpha \rho o v \sigma i \alpha ́ \sigma \varepsilon \omega v$ online $)$

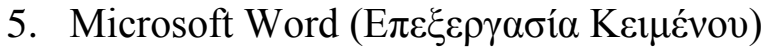

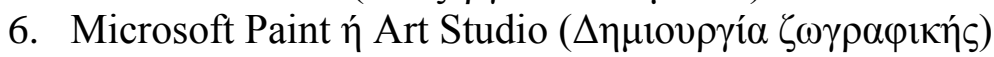

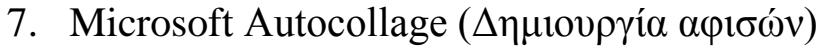

8. Microsoft DeepZoom ( $\Delta \eta \mu \imath$

SECTION B: applications, experiences, good practices, descriptions and outlines, educational activities, issues for dialog and discussion 
9. Microsoft Movie maker ( $\Delta \eta \mu$ iov $\gamma^{\prime i ́} \alpha$ videos $)$

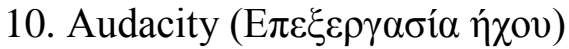

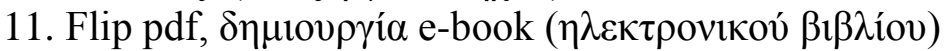

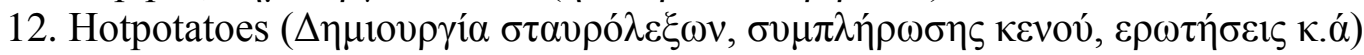

13. EclipseCrossword ( $\Delta \eta \mu 10 v \rho \gamma i ́ \alpha \sigma \tau \alpha \nu \rho o ́ \lambda \varepsilon \xi \omega v)$

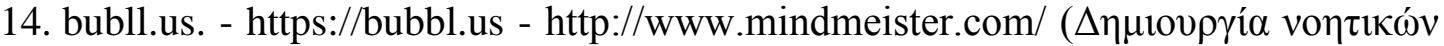

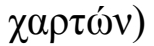

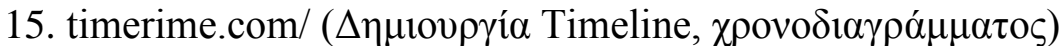

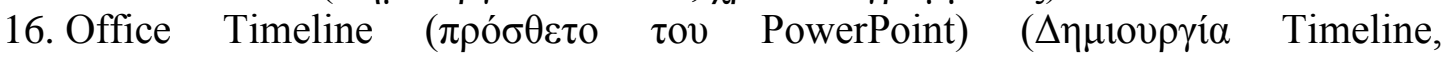

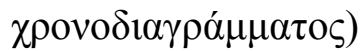

17. Jigsaw-puzzle http://www.tibosoftware.com/jigsaw-puzzle-free.htm $(\Delta \eta \mu 10 v \rho \gamma i ́ \alpha$ puzzles) $\eta$ http://www.jigsawplanet.com/ online puzzles

18. Ashampoo Photocomander 7

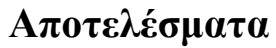

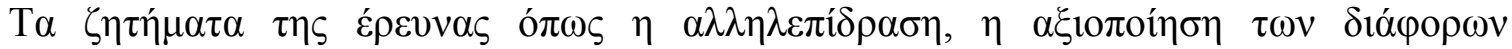

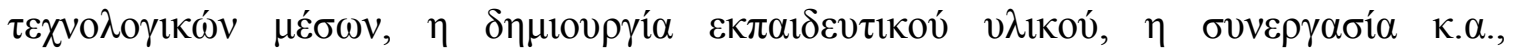

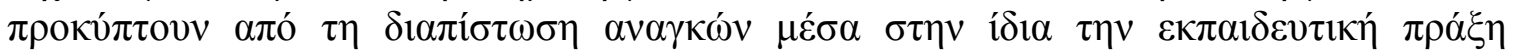

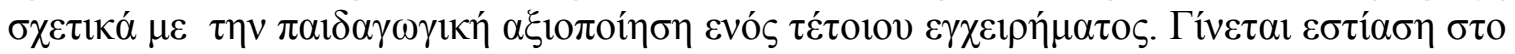

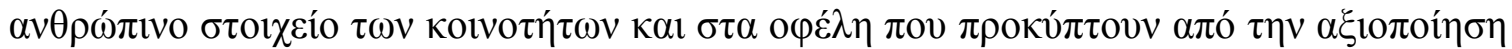

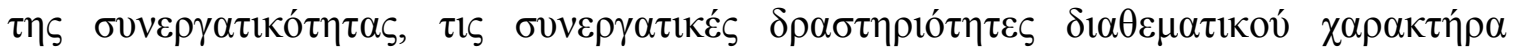

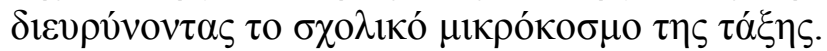

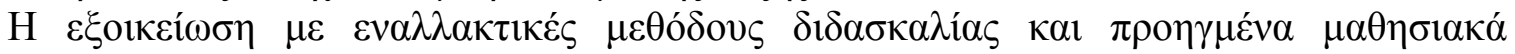

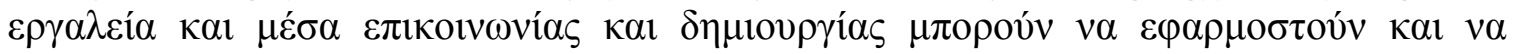

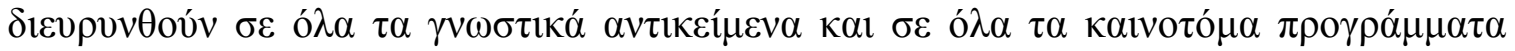

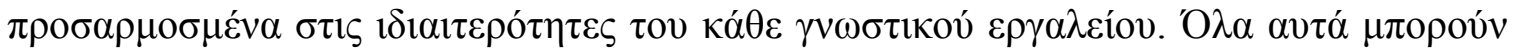

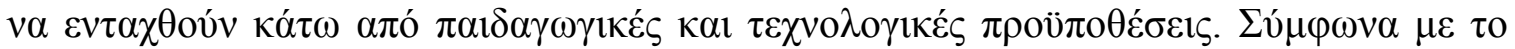

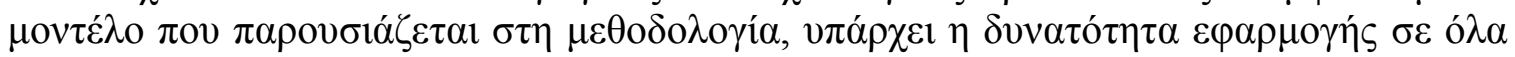

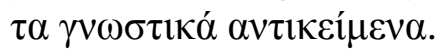

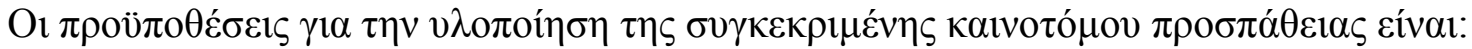

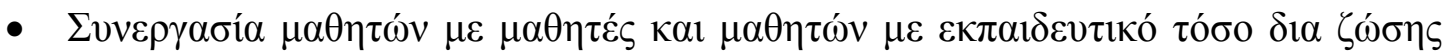

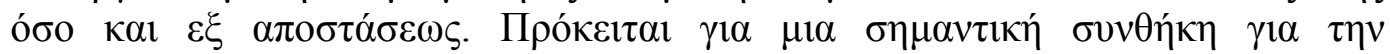

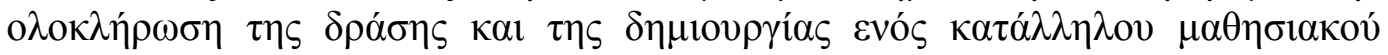

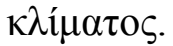

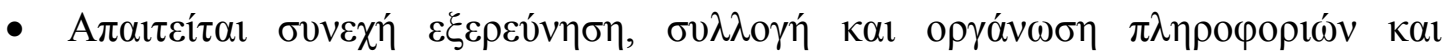
$\pi \varepsilon \rho 1 \varepsilon \chi 0 \mu \varepsilon ́ v o v$.

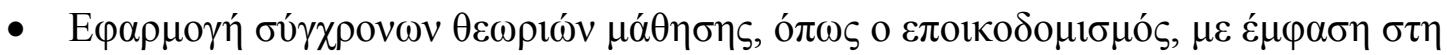

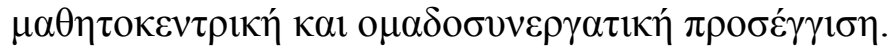

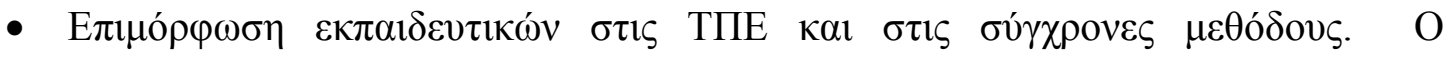

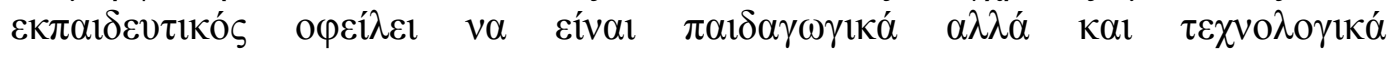

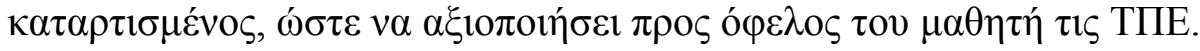

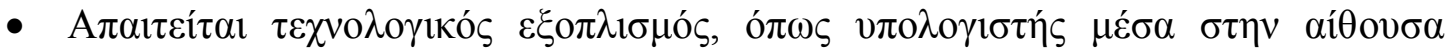
$\sigma v v \delta \varepsilon \delta \varepsilon \mu \varepsilon \dot{v} \sigma_{\varsigma} \mu \varepsilon \beta 1 v \tau \varepsilon \circ \pi \rho \circ \beta \circ \lambda \varepsilon \dot{\varepsilon} \alpha$.

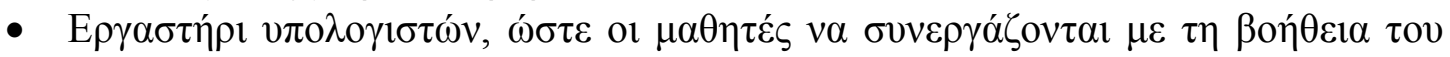

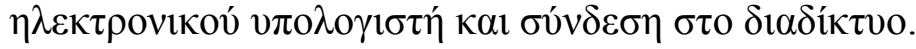

- A 


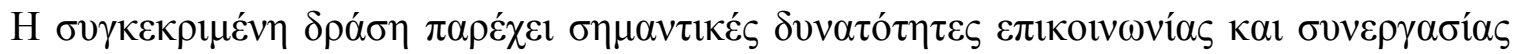

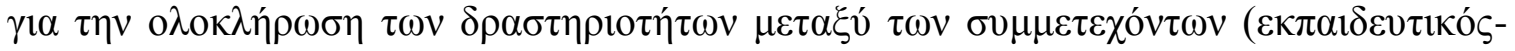

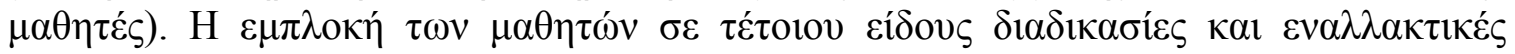

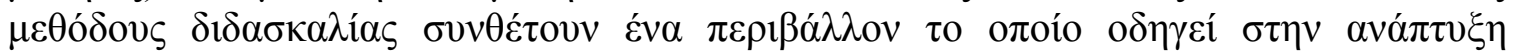

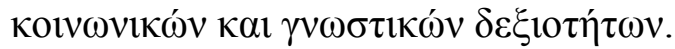

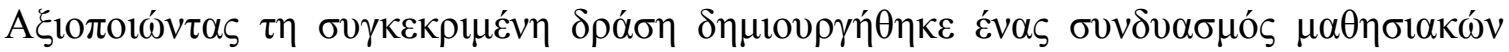

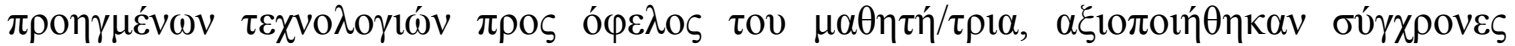

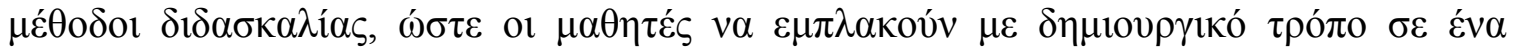

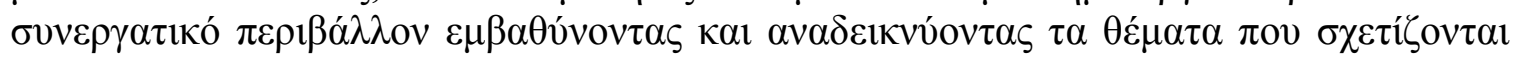

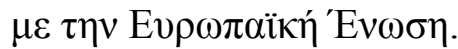

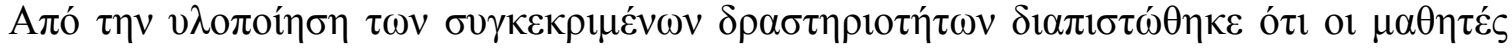

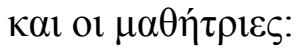

Е

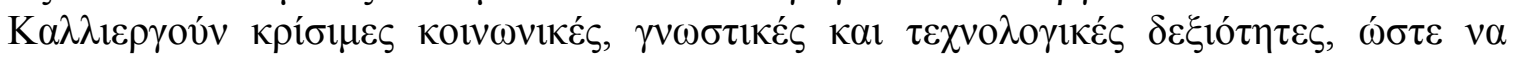

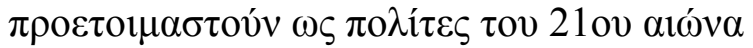

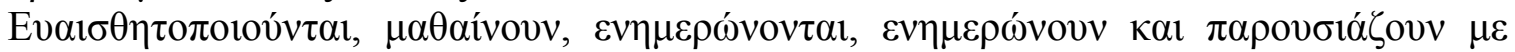

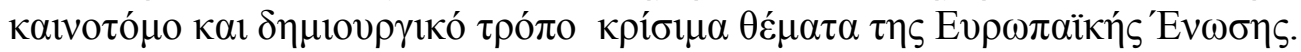

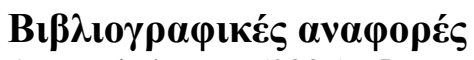

Anastasiades, P. (2009). Interactive Videoconferencing and Collaborative Distance Learning for K-12 Students and Teachers: Theory and Practice NY: Nova Science Publishers, Inc

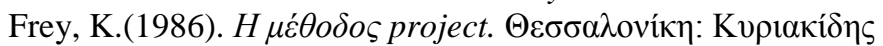

Galton, M. \& Williamson J. (1992). Group Work in the Primary Classroom. London: Routledge.

Swan, K., Shea, P., Dunne, E., \& Bennett, N. (1990). Talking and Learning in Groups. London: Macmillan.

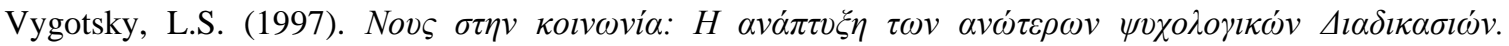

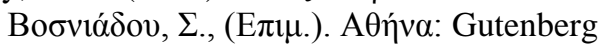

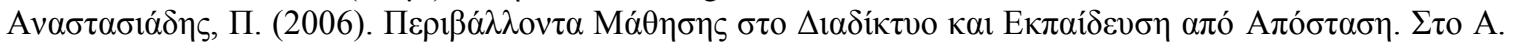

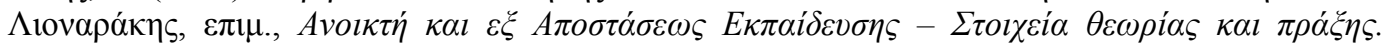
А $\theta$ v́va: Пролонло́

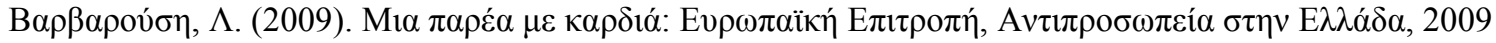

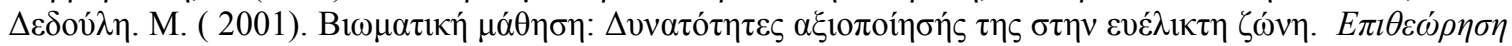

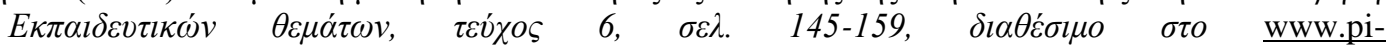
schools.gr/download/publications/epitheorisi/teyxos6/deloudi.PDF

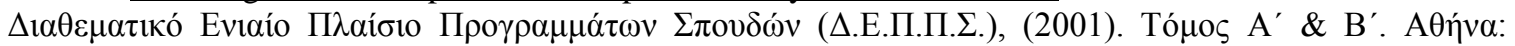

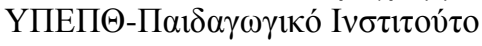

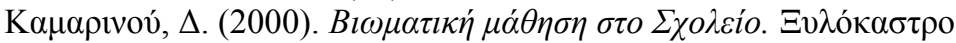

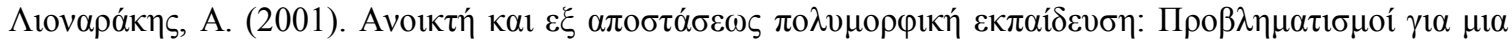

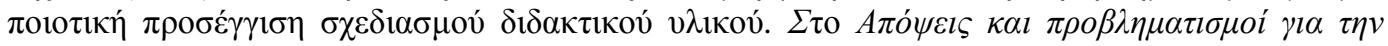

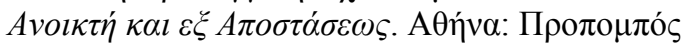

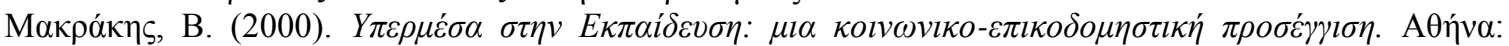

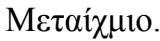

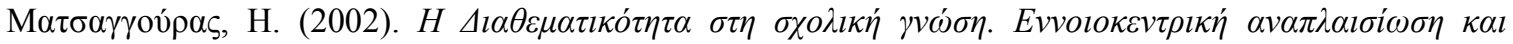

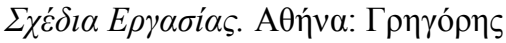

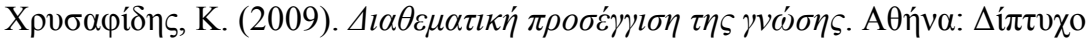

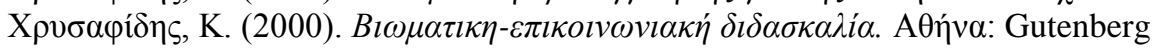

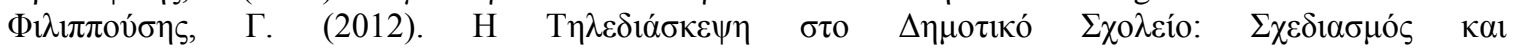

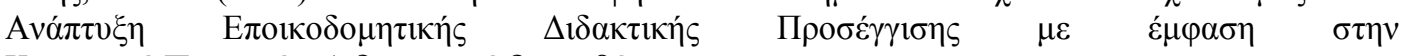

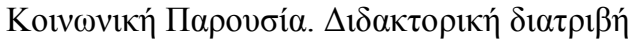

